\title{
Recommendations for accurate numerical blood flow simulations of stented intracranial aneurysms
}

\begin{abstract}
The number of scientific publications dealing with stented intracranial aneurysms is rapidly increasing. Powerful computational facilities are now available; an accurate computational modeling of hemodynamics in patient-specific configurations is, however, still being sought. Furthermore, there is still no general agreement on the quantities that should be computed and on the most adequate analysis for intervention support. In this article, the accurate representation of patient geometry is first discussed, involving successive improvements. Concerning the second step, the mesh required for the numerical simulation is especially challenging when deploying a stent with very fine wire structures. Third, the description of the fluid properties is a major challenge. Finally, a founded quantitative analysis of the simulation results is obviously needed to support interventional decisions. In the present work, an attempt has been made to review the most important steps for a high-quality computational fluid dynamics computation of virtually stented intracranial aneurysms. In consequence, this leads to concrete recommendations, whereby the obtained results are not discussed for their medical relevance but for the evaluation of their quality. This investigation might hopefully be helpful for further studies considering stent deployment in patient-specific geometries, in particular regarding the generation of the most appropriate computational model.
\end{abstract}

Keywords: cerebral aneurysms; CFD; hemodynamics; mesh dependency; turnover time; virtual stenting.

\footnotetext{
*Corresponding author: Gábor Janiga, Department of Fluid Dynamics and Technical Flows, University of Magdeburg "Otto von Guericke”, 39106 Magdeburg, Germany, Phone: +49 39167 18196, Fax: +49 39167 12840, E-mail: janiga@ovgu.de Philipp Berg and Dominique Thévenin: Department of Fluid Dynamics and Technical Flows, University of Magdeburg "Otto von Guericke", 39106 Magdeburg, Germany

Oliver Beuing and Martin Skalej: Department of Neuroradiology, University of Magdeburg “Otto von Guericke”, 39120 Magdeburg, Germany
}

Mathias Neugebauer, Rocco Gasteiger and Bernhard Preim:

Department of Simulation and Graphics, University of Magdeburg "Otto von Guericke”, 39106 Magdeburg, Germany
Georg Rose: Department of Healthcare Telematics and Medical Engineering, University of Magdeburg “Otto von Guericke”, 39106 Magdeburg, Germany

\section{Introduction}

In recent years, intracranial stenting has been proven to be an effective support tool in the endovascular treatment of ruptured and unruptured cerebral aneurysms $[4,15,17]$. Up to recently, the interventional approach was mostly confined to coiling alone. With the additional use of stents, a substantial number of aneurysms are now suitable for endovascular treatment, where surgical clipping was the only option in the past. This includes broadnecked aneurysms and cases in which the aneurysm neck incorporates important vessels without collateral flow.

It was soon noticed that intracranial stents might considerably reduce the flow entering the aneurysm sac [3]. Even complete thrombosis has been observed. This fact consequently led to the development of the so-called flow diverters, i.e., stents with a higher mesh density. With such devices, flow alteration is more effective and complete occlusion of aneurysms occurs more frequently as compared with conventional stent designs. In addition, no coiling is required. Leaving the vulnerable aneurysm sac itself outside the treatment strategy reduces the complications associated with the coiling procedure.

Nevertheless, treatment with flow diverters is still associated with known problems. Increased pressure values were detected after flow diverter deployment [11, 33], and as opposed to stents with conventional design, branching vessels should not be covered with this device. Otherwise, blood flow in these branches would be critically reduced, causing a high infarction risk within the tissue region supplied by these vessels. Nowadays, another drawback is the restriction to vessels with a sufficient diameter, for example, the internal carotid artery. Furthermore, the time to thrombosis seems in many cases to take up to several months, which is not sufficient in an 
emergency situation, i.e., for acutely ruptured aneurysms with a high risk of early rerupturing.

Thus, stents need to be developed that rapidly modify hemodynamics in a very circumscribed segment of the vessel (near the aneurysm ostium), while leaving the flow unchanged proximally and distally to the aneurysm and in nearby branching arteries. If available, such devices would yield a further decline in complication rates and an improvement of treatment success.

An accurate simulation of hemodynamics in the patient's geometry is required to choose and possibly develop the most appropriate patient-specific stent designs. Simultaneously, it would guide deployment strategies in order to improve aneurysm occlusion and avoid complications. Numerous studies concerning virtual stent investigations already exist in the literature, whereby refs. [39] and [40] are exemplarily mentioned here.

Under the auspices of the International Intracranial Stent Meeting, the Third Virtual Intracranial Stenting Challenge, abbreviated as VISC hereafter (http://bfc.ifs. tohoku.ac.jp/visc09/visc09_index.html), was announced in Sendai, Japan. The central objective of this VISC [31] was to assess the ability of engineering teams with expertise in computational hemodynamics in providing clinical partners with valuable pretreatment information within the time constraints of an elective endovascular intervention.

The authors of this work, the German research team MOBESTAN (German abbreviation standing for modeling and control of the flow behavior in aneurysms) at the University of Magdeburg "Otto von Guericke," has been selected as one of five groups worldwide presenting their results in Sendai. Two patient-specific datasets and the stent geometry have been provided by the organizers and employed to perform three-dimensional (3D) vascular geometry reconstruction, virtual stent deployment, and hemodynamic simulations using the given flow and stent information. As the number of groups dealing with stented intracranial aneurysms is rapidly increasing over the last years, the authors would like to share herewith their resulting experiences of a complete workflow from reconstructed image data to numerically generated flow fields and following analysis. Finally, recommendations can be derived in order to minimize the errors that can occur during such highly interdisciplinary investigations. In what follows, the first case provided by the VISC organizers will be used to illustrate all solution steps and encountered difficulties when virtually stenting a real patient-specific aneurysm geometry [20,21].

\section{Materials and methods}

The considered computational geometry is shown in Figure 1 before stent deployment. As explained previously, the corresponding dataset has been obtained from the reconstruction of real patient data provided by the VISC organizers. The provided STL data were of insufficient quality, and additional smoothing steps were necessary in order to generate highquality volume meshes in a later working step. The region of interest consists of two inlets and one outlet. Distal to the junction of both blood-feeding arteries, a saccular aneurysm with a mean diameter of approximately $9 \mathrm{~mm}$ is present.

\section{Computational geometry}

The dataset has been saved in the STL file format, which is the standard commonly used to store reconstructed geometries in image-based intracranial simulations. STL stands for stereolithography and is also often used for rapid prototyping. This format employs a set of unstructured triangles as surface description of a given volume.

The provided stent geometry has been deployed manually on a trial-and-error basis after deforming the vessel geometry, under the direct guidance of the medical partners involved in the project. On the basis of this experience and on many further tests, a semiautomatic virtual deployment procedure has been developed that has now been successfully validated [21]. The straight stent geometry prescribed by the VISC organizers has first been reproduced. To deploy the stent in the patient-specific geometry, the stent had to be scaled, bent, rotated, and moved in the desired position. Figure 2A shows the modified patient geometry obtained after deploying the stent (red wireframe) compared with the original configuration (opaque), as discussed in the following section.

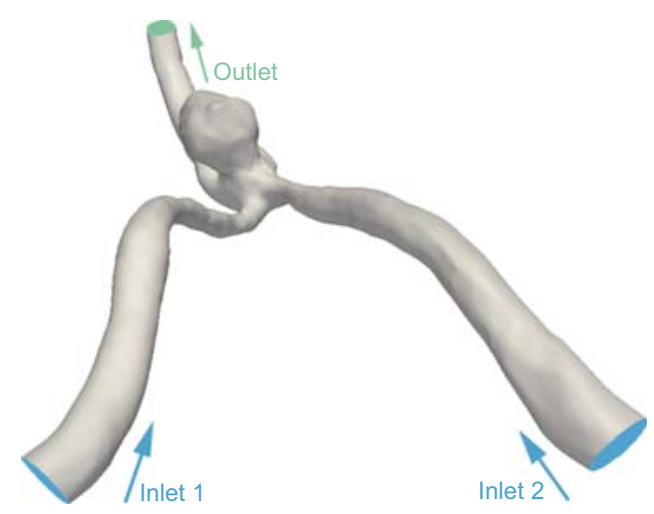

Figure 1 Original flow configuration of the first case provided for the VISC challenge [20]. 
A

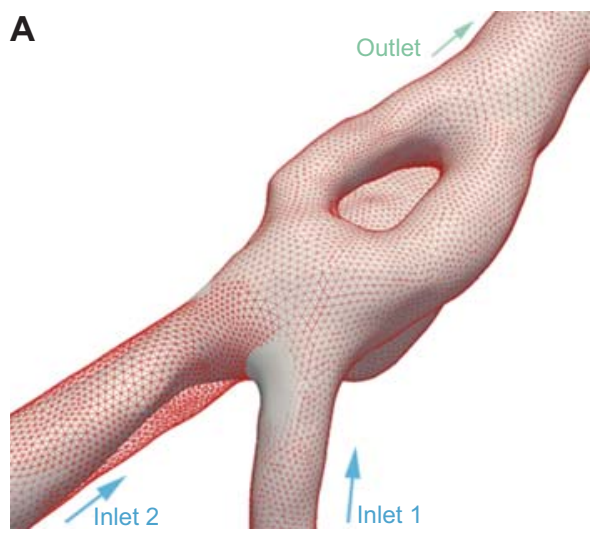

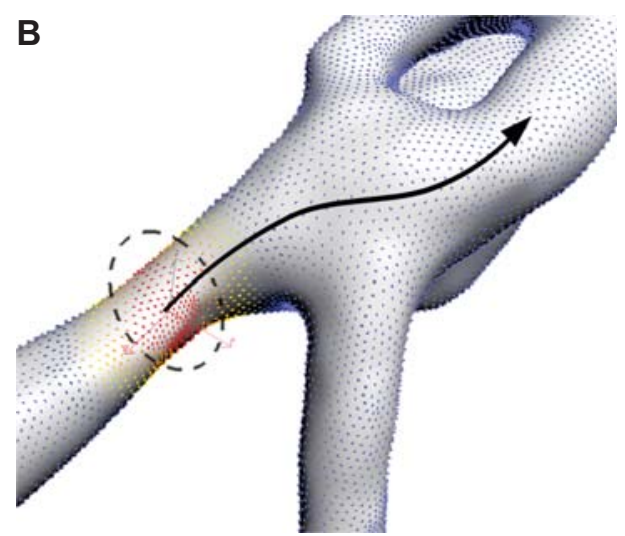

Figure 2 (A) Bottom view of the deformed patient geometry (red wireframe) on top of the original geometry (opaque) from Figure 1 after stent deployment. Surface optimization (as described in the section "Modification of the provided geometry") has already been performed. (B) Illustration of the final stent course (solid black line) and main deformation region (dashed black circle) resulting from an elastic, distance-based shape deformation of the vessel wall (red $=100 \%$, blue $=0 \%$ ).

\section{Modification of the provided geometry}

The provided surface geometry and stent are first modified during two very important preprocessing tasks: (1) improvement of the surface mesh quality and (2) geometry deformation associated with stent deployment.

\section{Improving surface mesh quality}

The provided geometries exhibit unsatisfactory quality for an accurate computational fluid dynamics (CFD) simulation.
The meshes suffer locally from strong inequilateral triangle shapes (e.g., acute-angled triangles) as illustrated in Figure 3A. Hence, the mesh quality has first been improved by systematically remeshing the surface using an advancing front mesh generator [34]. It employs a two-step approach to recreate the mesh. As an initial step, special points and edges are defined as invariable positions to steer the advancing front. For the considered application, it is particularly important to preserve the feature edges at the inlet and outlets.

Therefore, these edges are marked as fixed boundaries for the advancing front approach, based on their feature
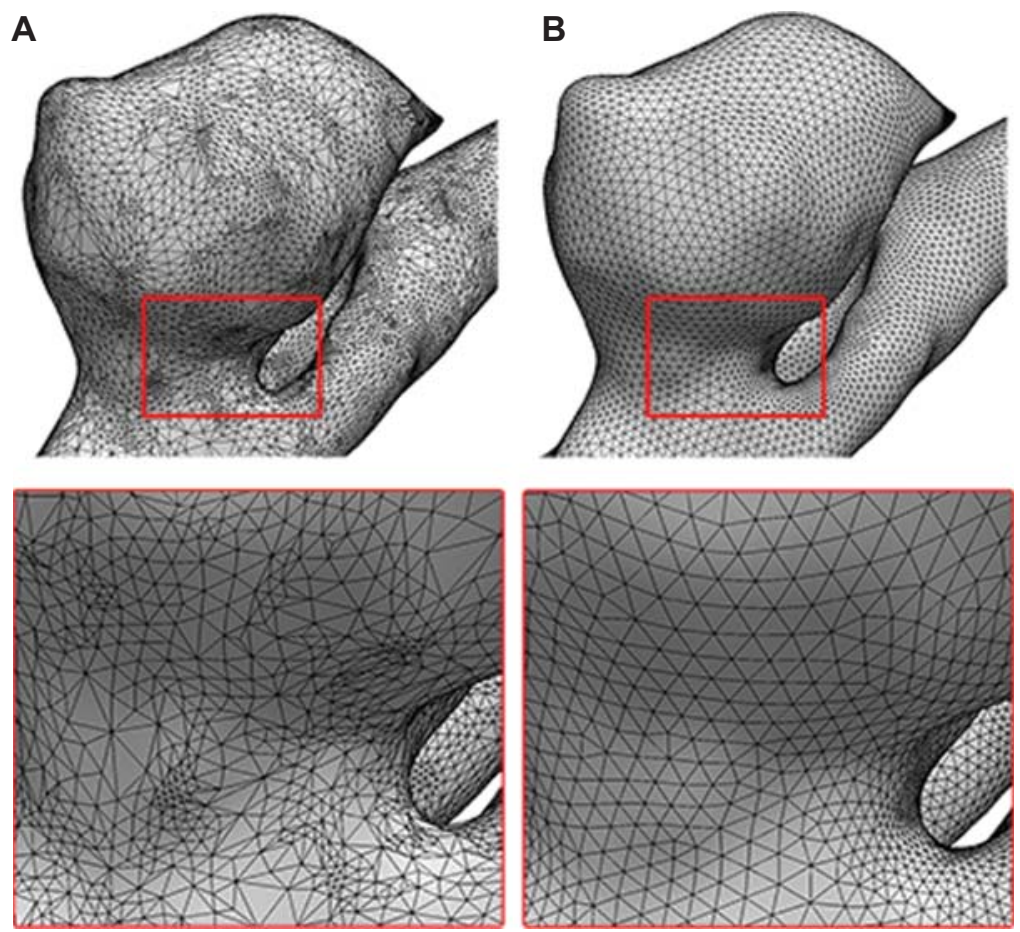

Figure 3 (A) Original, unsatisfactory mesh quality due to scalene triangle shapes. (B) Improved mesh quality after adaptive remeshing. 
angle. This feature angle is used to specify where surfaces will be broken by curves and vertices representing the maximum angle between mesh edges and faces [30]. The threshold for the selection must be selected appropriately to avoid an inclusion of sharp surface features, which would lead to an unwanted increase of the required mesh resolution. Surface features with a higher feature angle than the orthogonally cut inlets and outlets are most likely the result of reconstruction artifacts. They can be smoothed locally (e.g., with a Laplacian) to avoid their inclusion as boundary edges for the advancing front approach.

The size of the resulting mesh triangles depends on the local surface curvature and is adapted locally, delivering a good surface mesh with nearly equilateral triangles. Nevertheless, mesh quality can be improved even further, as done in a second step. A pointwise relaxation method, Broyden-Fletcher-Goldfarb-Shanno, of an angle/ area-based error function is employed to move the points toward the center of gravity defined by their neighbors. Topological changes such as edge swaps and point collapses are finally used. As a result, an excellent surface mesh quality is obtained (see Figure 3B), which is an absolute prerequisite for an excellent volumetric mesh [35]. It is important to point out that all improvements were carried out at the subvoxel level. Therefore, the changes are below the spatial resolution of the raw imaging data and the difference to the original surface is negligible.

\section{Reconstruction of the stent}

The stent geometry specified by the organizers of VISC representing a commercial Silk 4.0 flow diverter (Balt Extrusion, Paris, France) exhibited some inconsistencies regarding normal vector directions and vertex ordering. Therefore, the obtained mesh was not directly suitable for the application of the advancing front approach. Remeshing would have been, of course, possible. However, the stent graft is based on recurring patterns that can be easily parameterized, avoiding a computationally expensive, full remeshing. Instead, a reconstruction of the given stent geometry has been carried out using computer-aided design, allowing direct control over mesh quality, granularity, and feature edge handling.

\section{Geometry and mesh deformation}

Most published studies consider rigid walls during stent deployment (see, e.g., refs. [2, 7]). In contrast to significant vessel wall motions in the aortic arch, the radial dilatation of arteries in the circle of Willis does not increase $10 \%$ of the diameter [44]. Additionally, aneurysm initiation is caused, inter alia, by elastin degradation in the media and adventitia vessel layer. This leads to a decrease of flexibility and is one reason for the assumption of non-flexible walls in many numerical investigations. Additionally, there is no possibility to measure in vivo patient-specific intracranial vessel wall properties, which complicates the computational modeling. If the vessel flexibility should be considered within the numerical model, fluid-structure interaction simulations can be carried out. However, this is associated with a considerable increase in computational costs and can at present only rely on extremely simplifying assumptions such as isotropic material and constant wall thickness. Nevertheless, with increasing computational performance and more accurate measurements of wall properties, numerical simulations will certainly increasingly consider the radial deformation of vessels in the future.

In the present project, the involved clinical partners guided stent placement for a maximal therapeutic benefit based on their experience. Within the targeted area, the stent is initially aligned along the centerline of the vessel, computed using the polygonal model rather than the underlying image data. Thus, the accuracy of the resulting centerline depends on the local mesh resolution rather than on the image resolution (a meaningless quantity for the final CFD simulation). The embedded 3D Voronoi diagram is used to calculate the centerline [1]. This is the discrete solution for the continuous problem of finding the locus of centers of all maximum inscribing spheres. This type of centerline calculation leads to a very natural centerline pathway, even in pathological areas where the vessel differs from a tube-like morphology.

The stent is then placed and aligned along the centerline computed in this way. In stenotic areas near the ostium of the aneurysm, the stent leads to a dilatation of the vessel. The necessary deformation was prescribed manually in several iterations, steered by the experience of the clinical partners. This manual, expert-supported approach appears to be necessary in the absence of any reliably measurable information concerning patient-specific wall thickness and local wall properties that hinders a physically based computation of the deformation. Obviously, the resulting geometry is partly heuristic, which is always the case when exact material properties are not known. In the present case, the retained procedure has the advantage of building on top of practical experience based on hundreds of corresponding interventions by medical specialists. Figure 2B illustrates the resulting deformation of patient geometry. 
The finally retained stent pathway (solid black line with arrow in Figure 2B) leads from inlet 2 diagonally to the outlet. A local dilatation is applied to the stenotic area (dashed black circle in Figure 2B) of the inflow vessel proximal to the aneurysm, obviously affecting the resulting hemodynamics. A distance-based weighting for each vertex displacement (color-coded with red to blue in Figure 2B) has been finally applied, the weights being distributed according to a Gaussian function.

\section{Numerical mesh}

Figure 2A depicts the final configuration after stent deployment. The high-quality surface mesh obtained, thanks to the previously described procedure, can now directly be used for generating the volume mesh required for CFD. Mesh generation was carried out using the commercial software ANSYS ICEM CFD V12.0.1 (Ansys Inc., Canonsburg, PA, USA). The resulting volume mesh quality has been quantified based on the equiangle as well as on the equivolume skewness, two standard criteria for CFD [10]. A volume mesh always has a much lower quality than the associated surface mesh. Therefore, the excellent quality of the surface mesh is a crucial step in mesh generation, as the quality of the final volume mesh noticeably influences the numerical results, as shown in what follows. An insufficient mesh quality may lead to convergence problems or, even worse, to a completely wrong solution.

The easiest and in practice widely used mesh type for such a complex geometry is a pure tetrahedral mesh. Many automatic meshing tools are available to create such an unstructured mesh. If this volume mesh is sufficiently fine, it may indeed accurately predict the fluid flow. However, a previous study of the authors has shown that the wall shear stress (WSS) distribution cannot be predicted accurately in this manner even using a very fine and well-resolved mesh [29]. An additional, fine prism mesh is absolutely required near the walls as commonly used in, e.g., external aerodynamics. This drawback of the pure tetrahedral mesh has also been reported in other numerical simulations of intracranial aneurysms $[6,19]$.

As a consequence, one- and three-layer-prism meshes have been built systematically along the wall, leading to an average elevation from the first mesh element to the wall of around $20 \mu \mathrm{m}$. In case of the investigation of transitional flows, an issue not considered here, it would be recommended to further refine the boundary layer. Obtaining such a fine resolution is hardly possible using only tetrahedral cells without an excessive skewness quality problem. However, the core region of the domain can be indeed meshed by tetrahedral, finite volume cells. As a first summary, the choice of mesh type depends on the flow variables that are in the center of interest. For properties along the vessel walls (e.g., pressure, WSS), prism layers are mandatory. However, the investigation of core flow structures such as vortices or helixes can be carried out using pure tetrahedral grids, if the resolution is fine enough to achieve mesh-independent solutions.

The virtually stented configuration has been finally discretized with up to a total number of $6,985,877$ cells for case $7 \mathrm{M}$-tetra (Table 1). Figure 4 shows a cut through the aneurysm sac, where the finer mesh indicates the stent location.

Table 1 Number of finite volume cells and mesh quality considered in the computations.

\begin{tabular}{lrrrr}
\hline Case & No. of cells & $\begin{array}{r}\text { Prism } \\
\text { layers at } \\
\text { the wall }\end{array}$ & $\begin{array}{r}\text { Max. } \\
\text { equivolume } \\
\text { skewness }\end{array}$ & $\begin{array}{r}\text { Max. } \\
\text { equiangle } \\
\text { skewness }\end{array}$ \\
\hline No stent & $4,360,896$ & 0 & 0.800 & 0.819 \\
0.5M & 535,325 & 0 & 0.998 & 0.991 \\
2M-p1 & $1,983,938$ & 1 & 0.918 & 0.862 \\
2M-tetra- & $1,726,582$ & 0 & 0.933 & 0.892 \\
uniform & & & & \\
2M-tetra- & $1,667,001$ & 0 & 0.900 & 0.902 \\
non-uniform & & & & \\
5M-p3 & $4,590,290$ & 3 & 0.905 & 0.914 \\
5M-tetra & $4,785,186$ & 0 & 0.934 & 0.904 \\
7M-tetra & $6,985,877$ & 0 & 0.836 & 0.875 \\
7M-p3 & $6,939,966$ & 3 & 0.853 & 0.853 \\
\hline
\end{tabular}

The case names contain roughly the number of elements in million $(M)$ and indicate whether prism layers ( $p$ ) were considered.

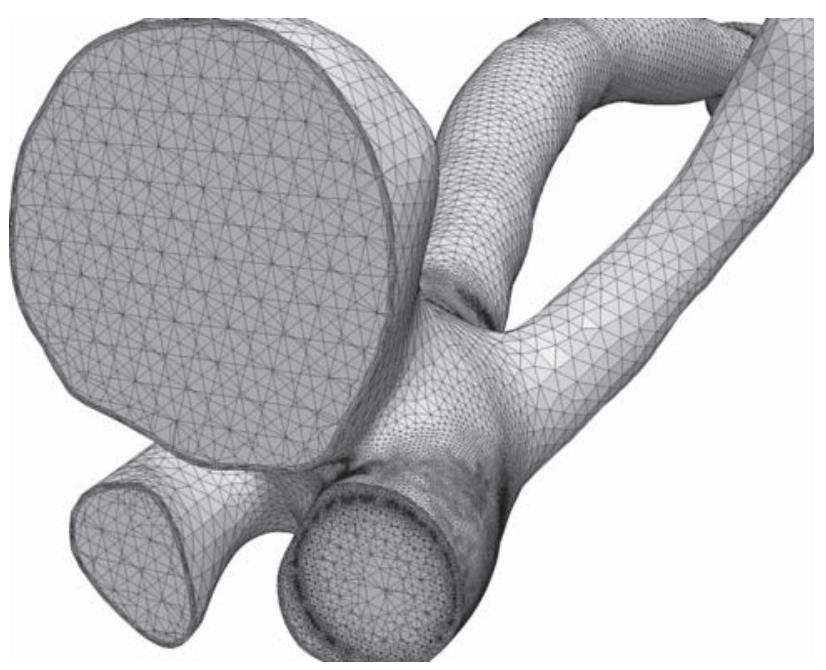

Figure 4 Zoom on the volume mesh showing the fine mesh structure in the vicinity of the wall and of the stent wires. The prism layers can be clearly observed in the near-wall region. 


\section{Hemodynamic simulations}

Blood rheology is represented using a Newtonian description with constant density and viscosity, where blood density is chosen as $1000 \mathrm{~kg} / \mathrm{m}^{3}$ and dynamic viscosity as $4 \times 10^{-3} \mathrm{~Pa} \cdot \mathrm{s}[31,38]$. Previous works have demonstrated that using a more realistic, non-Newtonian fluid model should not be necessary for the conditions considered in the present configuration $[8,18]$, as it involves only large vessels $(>0.5 \mathrm{~mm}$ ) and comparatively low shear rates. However, other groups found that this might not always hold in general, and appropriate rheological models, e.g., Bingham or Carreau-Yasuda, may be needed even for this range of vessel diameter $[13,16]$. Because such models are computationally tractable, it is expected that non-Newtonian models will become the rule in the near future.

\section{Inlet boundary conditions}

At the inlet of the computational domain, the velocity is very often prescribed as a Dirichlet boundary condition (prescribed values). Corresponding velocity values can be measured, e.g., by Doppler ultrasound or phase-contrast magnetic resonance angiography. For most of the published numerical studies, this information was not directly accessible. Therefore, only estimations based on integrated quantities or on the literature are employed, often combined with the assumption of a constant, uniform velocity profile. This very simple situation might, however, lead to misleading interpretations [27]. For instance, the computed WSS values in the vicinity of the inlet are completely meaningless, as a uniform velocity profile theoretically leads to an infinite, local WSS (or, in practical CFD, to extremely large values).

To overcome this issue, the inlet cross section has been extracted from the considered computational domain and a separate channel flow has been simulated in a preprocessing step. Only a short channel is considered and meshed; however, the two ends of this section are connected using a periodic boundary condition, modeling an infinite channel flow with a uniform cross section and a prescribed mass flow rate. Obviously, the position of this inlet should be chosen in a suitable way, sufficiently far from any strong bend. At the end of the preprocessing step, a fully developed velocity distribution is obtained and implemented as fixed boundary condition for the further CFD of the flow in the patient geometry, eliminating the problem mentioned previously.

Note that, in the present publications, only steady flow conditions are discussed. The authors are fully aware that pulsatile flows are more realistic and more interesting in principle. The pulsatile nature of the blood flow has been considered in many other publications of our group $[5,6,8,41]$ and is particularly important to compute rupture indicators. In contrast, many publications have demonstrated that averaging pulsatile CFD results leads to very similar results to those obtained - at a much lower computational cost - by steady CFD [6, 41]. In the present case, a steady flow condition has been finally retained with an inlet flow rate of $2.36 \times 10^{-6} \mathrm{~m}^{3} / \mathrm{s}$ at each inlet, as prescribed by the VISC organizers. Owing to the different cross sections, this leads to mean velocity values of 0.2045 and $0.1102 \mathrm{~m} / \mathrm{s}$ at the first and second inlet, respectively. The Reynolds number at the outlet of the considered geometry is 470 based on the mean velocity and hydraulic diameter, showing that the steady flow is laminar and eliminating the need for any turbulence model.

All vascular walls are assumed to be rigid, as done in most published studies (see, for instance, ref. [37] and the cited references in this review), as real wall material properties are completely unknown and highly patient dependent. A standard, no-slip boundary condition (i.e., zero velocity for rigid walls) is employed at all contact points with surfaces (vessels, stent).

\section{Outlet boundary condition}

At the outlet, either resistance or traction-free boundary conditions are usually applied. The resistance model is described by $p=R \cdot q$, where $R$ denotes the resistance and $q$ the mass flux. A vascular bed model can be used to evaluate the resistance $R$ [26]. This would be particularly useful if many outlets are considered with widely varying geometric properties, or if the pressure field is of particular interest.

The traction-free condition prescribes directly the pressure at the outlet. In this study, only one outlet is considered. Hence, both boundary conditions deliver the same results for a steady flow. As a consequence, the outlet boundary condition has been simply chosen as a prescribed, uniform relative pressure of $0 \mathrm{~Pa}$.

\section{Case descriptions}

All simulations have been carried out on the Kármán Linux cluster equipped with 68 dual nodes [Quad-Core AMD Opteron 64-bit Processor 2352 (AMD, Sunnyvale, CA, USA) with $2.1 \mathrm{GHz}$ clock rate] using a high-speed InfiniBand interconnection. The numerical computations are performed in parallel using up to eight computing cores applying the 
commercial CFD solver ANSYS-Fluent V12.0.1. As explained previously, the mesh quality is essential for accurateresults. Getting correct predictions is of course a key requirement if the results should be used to guide treatment options considered by the medical doctors. As a consequence, several different meshes have been compared in this work, as described in Table 1, containing also the number of prism layers along the artery wall (indicated with the letter $p$ followed by the number of prisms layers). The figure preceding $M$ in the case name gives roughly the number of computational cells, expressed in millions. The first case correspond to the patient geometry without stenting, all other cases contain the stent placed as shown in Figure 5. The employed body-conforming grids involve roughly between 1 and 7 million finite volume cells. The largest mesh with 7 million elements requires a total amount of $10 \mathrm{~GB}$ of memory applying a double-precision solver on a 64-bit system. The computation with this particular mesh is realized within $<7 \mathrm{~h}$ wall clock time using second-order discretization and reaching normalized residuals of $10^{-8}$ when using eight computing cores in parallel.

\section{Analysis}

Note that, for the sake of brevity, generic issues associated with flow simulations of cerebral aneurysms are not discussed in the present article. The interested reader is referred, for instance, to the review of Sforza et al. [37] and to further publications for corresponding information [11, 12, 28, 41].

The numerical flow simulation provides various hemodynamic quantities. After a first qualitative examination relying on contour or vector plots of the velocity field, the modification of key hemodynamic parameters is analyzed quantitatively in what follows. For the sake of completeness, not only the commonly investigated WSS has been studied in detail, as it is still unclear how this parameter may correlate with the risk of rupture; as an important additional quantity, the inflow rates at the aneurysm neck as well as the stasis in the aneurysm have also been investigated, as they mainly determine the thrombosis rate within the aneurysm. The flow stasis has been computed through the turnover time, as it has been done in ref. [22] for idealized and patient-specific geometries. A successful stenting treatment should change the hemodynamics in the aneurysm and produce thrombogenic conditions, i.e., reduce the flow velocity and elongate the stasis. To stimulate the aneurysmal thrombosis, the increase of the stasis in the aneurysm should thus be specifically targeted [25]. Previous studies $[9,23,45]$ have already shown that increasing aneurysmal flow turnover time supports thrombus formation in cerebral aneurysms. Considering all these results, the turnover time is used as the major indicator of stasis [24] in the present work. Clearly, quantities derived from unsteady simulations would be certainly essential to more accurately quantify the risk of rupture. This is, however, not the focus of the present study.

\section{Results}

Before statements about the hemodynamic effects of the stenting procedure can be made, the impact of the most important numerical settings has been checked in a systematic manner:

1. Single-precision vs. double-precision computations: case 5M-p3 has been computed with both a singleand a double-precision solver, on either a 32-bit and a 64-bit system, yielding four results. Surprisingly, all relevant hemodynamic variables are found to be within $1 \%$ of each other. As a consequence, singleprecision simulations are recommended, as they are considerably faster.

2. All computations have been performed applying either first- or second-order accurate numerical discretization

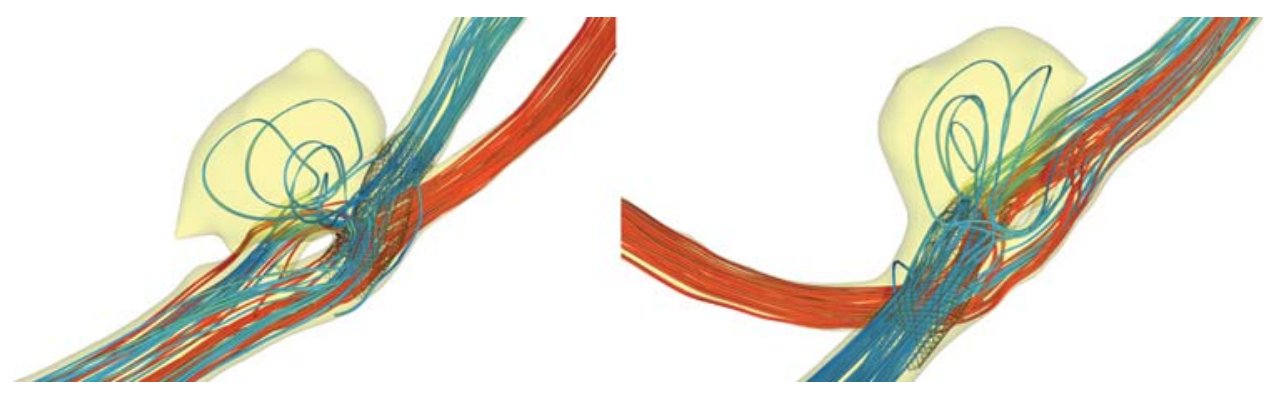

Figure 5 Two representations of the virtual stent (black wireframe) in the patient geometry after deformation, together with selected streamlines (red from inlet 1 , blue from inlet 2) in the stented geometry for case 5M-p3. 
schemes. It has been observed that the peak velocity value within the aneurysm sac might show a $>15 \%$ relative difference between a first-order and a secondorder computation, with all other parameters and grid being identical. Therefore, second-order computations are highly recommended whenever available.

3. Different residual reduction values have been also prescribed to detect convergence. Results obtained with ANSYS CFX and with the open source solver OpenFOAM (although not discussed in the present work in the interest of space) have been involved as well in corresponding studies. From this analysis, it can be stated that the default settings of most CFD solvers are often not a sufficient constraint. Considering the present experience, residual reduction should be at least a factor $10^{5}$ for the mass conservation equation (continuity). This is normally enough to ensure simultaneous convergence for all other flow quantities in a steady, laminar, incompressible case.

Then, the mesh dependence of the numerical results has been carefully checked when including the stent. The first mesh with $<1$ million finite volume cells (case $0.5 \mathrm{M}$ ) is not included in further analysis because it was impossible to obtain a converged flow solution. This is not a real surprise but a direct consequence of an inappropriate mesh quality (see quality parameters in the second row of Table 1).

In one previous study of the authors, the effect of the numerical mesh has been examined comparing only the WSS distribution [29] and choosing the finest mesh as a reference grid. In the present case, this finest mesh involves almost 7 million finite volume cells (last row in Table 1). This was shown to be sufficient and lead to mesh-independent results in ref. [6]. Here, the impact of mesh type (structured vs. unstructured), solver (Fluent vs. OpenFOAM), and time dependency (steady vs. unsteady) was investigated comparing hemodymically relevant parameters such as velocity, pressure, WSS, and turnover time. Regarding mesh dependency, it turned out that noticeable changes occur only up to 5 million elements.

The computation with a second-order upwind discretization scheme performed for case $7 \mathrm{M}$-p3 is again retained here as a reference solution, and all the other numerical simulations are compared with this result. Additionally, two different tetrahedral meshes have been investigated for 2 million cells. The uniform case (2M-tetra-uniform) relies on a uniform mesh throughout the cross section, while the non-uniform mesh (case 2M-tetra-non-uniform) is refined close to the wall, leading to relatively large volume elements in the core region.
The computational results for the velocity and the hemodynamic parameter WSS are exemplified in Figure 6. Here, the isovelocity surfaces (A and B) correspond to 10 $\mathrm{cm} / \mathrm{s}$ and illustrate the effect of stent deployment. Also, the WSS was significantly decreased after stenting ( $\mathrm{C}$ and D). For more information about the importance of WSS distribution in intracranial aneurysms, refs. [42, 43] are recommended.

A quantitative analysis is now needed to quantify the impact of stent deployment. Unfortunately, no general agreement can be found in the literature about the most relevant criterion for cerebral aneurysms. Morphological properties have been extensively analyzed, e.g., in refs. $[14,32]$. Hemodynamic characterization was the subject of the work published by Burleson and Turitto [9], considering in particular hemodynamic shear stress and turnover time of blood within the aneurysmal sac. Both morphological and hemodynamic parameters were investigated in ref. [46] for $>100$ cases. In the present study, only one of the two provided patient geometries is considered, as prescribed by the VISC organizers. Therefore, the emphasis is set only on hemodynamics in what follows.

Several authors stated that excessive WSS might cause rupture, whereas others consider that low values are dangerous and should be eliminated. Considering that the emphasis is placed here more on the impact of the computational procedure, only the peak WSS values are listed in Table 2. Indeed, the employed computational meshes lead to considerable variations of this property.

Additionally, the flow stasis within the aneurysm has been computed from the turnover time [22, 36] and is shown in Table 2 as well. This turnover time is determined by dividing the full aneurysm volume by the inlet volume flow rate found at the aneurysm neck, defined as a straight plane placed directly above the stent. The aneurysmal inflow rate is obtained by integrating the entering flow rate over the full cross section.

Finally, the maximum and the mean values of the velocity magnitude within the aneurysm sac have been listed as well in Table 2.

\section{Discussion}

Considering the results compiled in Table 2, it is clear that all numerical grids with $<3$ million cells underpredict the turnover time by $>10 \%$, except when a suitable local refinement is employed. Cases 5M-p3 and 5M-tetra (5 million cells) are in acceptable agreement with the reference result concerning this quantity, with $<5 \%$ difference. 


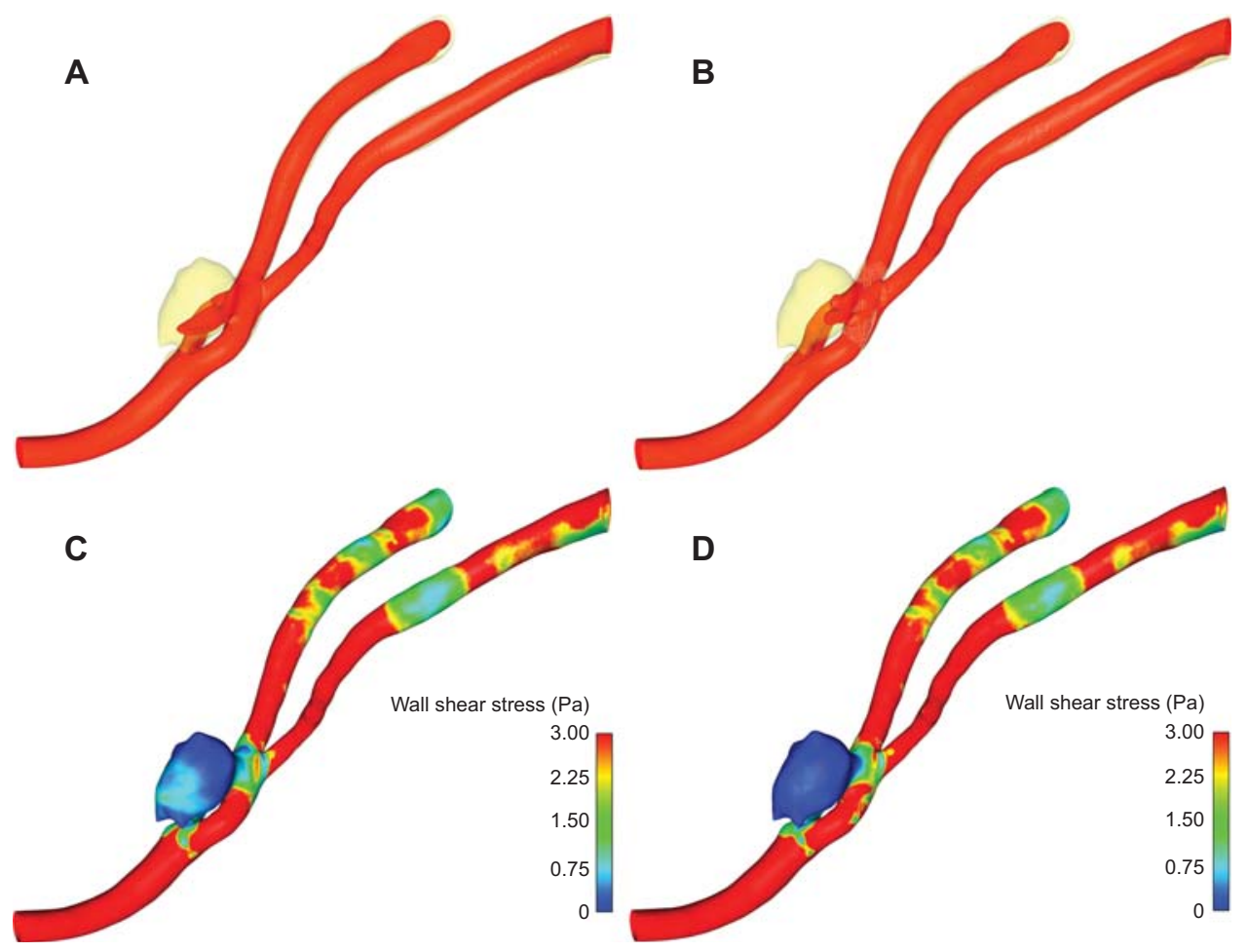

Figure 6 Velocity and WSS reduction due to stent deployment (case 7M-p3). Isosurface velocity and WSS distribution before (A, C) and after stenting (B, D).

Only the meshes with prism layers are able to determine the WSS values with an accuracy comparable to that of the reference mesh. Cases 2M-p1 and 5M-p3 show an error in peak WSS below 5\% compared with the reference solution. All computations without a prism layer significantly overpredict the maximum WSS values. Combining both criteria, only case 5M-p3 appears to predict both key quantities with a precision level comparable to the reference mesh but at a noticeably reduced computational cost.

Concerning stenting efficiency, the inflow rate exchanged between the main vessel and the aneurysm is reduced to $60 \%$ of its original value after stenting, leading to a corresponding increase of the turnover time by the same factor. The hemodynamic alteration is hence successful in the present case when applying the chosen stent design in the manner described previously. A similar modification (reduction by about a factor 2) is observed for the average and maximum velocity within the aneurysm sac (Table 2).

It is now possible to summarize the main findings of the present study as follows:

1. The importance of an accurate reconstruction of the patient vessel geometry is obvious in order to obtain useful results when deciding on further specific treatment options.

Table 2 Quantitative comparison of inflow rate, turnover time, and maximum WSS in the aneurysm sac for all meshes.

\begin{tabular}{|c|c|c|c|c|c|c|c|c|c|}
\hline Case & $\begin{array}{r}\text { Inflow rate } \\
\left(\mathrm{cm}^{3} / \mathrm{s}\right)\end{array}$ & $\begin{array}{r}\text { Turnover } \\
\text { time (s) }\end{array}$ & $\begin{array}{r}\text { Rel. diff. to } \\
7 M-p 3\end{array}$ & $\begin{array}{c}\text { Maximum } \\
\text { WSS }(\mathrm{Pa})\end{array}$ & $\begin{array}{r}\text { Rel. diff. to } \\
7 M-p 3\end{array}$ & $\begin{array}{r}\text { Average } \\
\text { vel. }(\mathrm{cm} / \mathrm{s})\end{array}$ & $\begin{array}{r}\text { Rel. diff. to } \\
7 M-p 3\end{array}$ & $\begin{array}{r}\text { Peak vel. } \\
(\mathrm{cm} / \mathrm{s})\end{array}$ & $\begin{array}{r}\text { Rel. diff. to } \\
7 M-p 3\end{array}$ \\
\hline No stent & 0.4456 & 0.646 & $-37.94 \%$ & 6.507 & $-24.02 \%$ & 3.400 & $88.89 \%$ & 37.37 & $80.53 \%$ \\
\hline $2 M-p 1$ & 0.3043 & 0.944 & $-9.32 \%$ & 9.205 & $7.48 \%$ & 1.605 & $-10.83 \%$ & 20.07 & $-3.04 \%$ \\
\hline 2M-tetra uniform & 0.3124 & 0.919 & $-11.72 \%$ & 11.486 & $34.12 \%$ & 1.820 & $1.11 \%$ & 16.76 & $-19.03 \%$ \\
\hline 2M-tetra-non-uniform & 0.2751 & 1.046 & $0.48 \%$ & 13.525 & $57.93 \%$ & 1.605 & $-10.83 \%$ & 16.26 & $-21.45 \%$ \\
\hline $5 M-p 3$ & 0.2766 & 1.037 & $-0.38 \%$ & 8.755 & $2.23 \%$ & 1.722 & $-4.33 \%$ & 19.43 & $-6.14 \%$ \\
\hline $5 \mathrm{M}$-tetra & 0.2845 & 1.008 & $-3.17 \%$ & 9.797 & $14.40 \%$ & 1.666 & $-7.44 \%$ & 18.49 & $-10.68 \%$ \\
\hline $7 \mathrm{M}$-tetra & 0.2716 & 1.058 & $1.63 \%$ & 8.928 & $4.25 \%$ & 1.696 & $-5.78 \%$ & 20.16 & $-2.61 \%$ \\
\hline $7 M-p 3$ & 0.2758 & 1.041 & Ref & 8.564 & Ref & 1.800 & Ref & 20.70 & Ref \\
\hline
\end{tabular}


2. The quality of the geometric reconstruction directly conditions the quality of the surface grid and will hence strongly affect the quality of the volume grid. As this parameter controls in turn the key hemodynamic parameters, the preprocessing steps (geometric reconstruction, generation of a surface grid) must be carried out with great care.

3. As long as vessel and aneurysm vessel properties cannot be measured locally and in a patient-specific manner with an acceptable accuracy, it seems to be reasonable to assume non-flexible walls due to the mentioned factors. However, wall elasticity has an influence on the hemodynamics and has to be studied in the future.

4. Because no significant differences have been observed, single-precision simulations are recommended as they are considerably faster than double-precision runs.

5. Concerning discretization, considerable differences are observed. A second-order discretization is recommended, in particular for relatively coarse meshes. The impact of the order is as expected reduced for very fine grids.

6. Numerical tests reveal that a residual reduction by a factor of $10^{5}$ is necessary and sufficient to reach the needed convergence level.

7. By retaining simultaneously the turnover time, the peak velocity, and the peak WSS in the aneurysm sac as key hemodynamic quantities, a relatively coarse computational mesh (2M-p1, coarsest acceptable grid resolution) can still be used to predict all resulting flow conditions with an accuracy $<20 \%$. When an accuracy $<10 \%$ is needed, 5 million volume elements should be used. At least a few prism layers are absolutely necessary if WSS must be accurately predicted, but are superfluous for the other listed quantities.

Note that some remaining issues cannot be tested in the present configuration. For the considered case, prescribing suitable inflow and outflow boundary conditions is relatively straightforward, as described previously. The issue becomes much more complex when several outflows have to be taken into account simultaneously. Only steady-state computations have been presented, as defined by the VISC organizers. Even if many publications tend to show that unsteady simulations do not lead to very different results, this might not apply for all conditions. As unsteady simulations are needed to compute most rupture indicators, unsteady simulations are considered in many other projects $[8,41]$. Considering blood as a simple Newtonian fluid should be applicable for the present geometry but might certainly be unsuitable for other geometries involving much smaller vessels or when trying to simulate the progress of thrombosis.

\section{Conclusions}

In this article, the impact of the computational settings on the CFD results has been investigated considering a stented aneurysm based on real patient data. Geometry deformation has been applied in order to deploy the stent in a realistic manner. The quality of the surface mesh has been first improved to finally produce high-quality volume meshes, as required for computations that should ultimately support medical treatment options.

All the steady-state simulations discussed in this publication have been performed using the commercial flow solver ANSYS-Fluent V12.0.1. The computational results have been compared before and after deploying the virtual stent. Both qualitative and quantitative analyses have been employed to predict the effect of the virtual stent deployment and the influence of the mesh and of all numerical parameters. It has been found that the stent deployment increases the turnover time in the aneurysm by a factor of almost 2. It is believed that this modification should enhance thrombus formation. A sufficiently fine mesh is necessary to resolve the stent and to obtain accurate CFD estimations, depending on the acceptable error level.

Finally, recommendations have been proposed concerning CFD for virtual stenting applications, covering a variety of issues from surface mesh to boundary conditions and from second-order discretization to residual values at convergence.

Acknowledgments: This work was financially supported in part by the International Max Planck Research School for Analysis, Design and Optimization in Chemical and Biomedical Process Engineering Magdeburg and the Land Saxony-Anhalt in Germany. The help of S. Seshadhri to produce the CAD geometry of the stent and to support the mesh generation is highly acknowledged. The permission to publish a case from the VISC challenge has been provided by the main organizer, Professor Makoto Ohta, and is warmly acknowledged.

Conflict of interest statement: We declare that we have no conflict of interest.

Received December 21, 2012; accepted April 3, 2013 


\section{References}

[1] Antiga L, Steinman DA. Robust and objective decomposition and mapping of bifurcating vessels. IEEE Trans Med Imag 2004; 23: 704-713.

[2] Appanaboyina S, Mut F, Lohner R, Putman C, Cebral J. Simulation of intracranial aneurysm stenting: techniques and challenges. Comput Methods Appl Mech Eng 2009; 198: 3567-3582.

[3] Augsburger L, Reymond P, Rufenacht DA, Stergiopulos N. Intracranial stents being modeled as a porous medium: flow simulation in stented cerebral aneurysms. Ann Biomed Eng 2011; 39: 850-863.

[4] Baptista T, Fragata I, Ribeiro C, Reis J. Flow diverters devices for treatment of intra-cranial aneurysms - six months follow-up results. Acta Med Port 2012; 25 Suppl 1: 13-16.

[5] Berg P, Janiga G, Thévenin D. Instationäre Strömungssimulationen und Auswertung von Blutströmungen in zerebralen Aneurysmen mit Stent. In: Burgert O, Schipper J, Zachow S, editors. Computer- und Roboterassistierte Chirurgie (CURAC). Magdeburg, Germany: ISBN 978-3-940961-59-4 2011: 91-94.

[6] Berg P, Janiga G, Thévenin D. Detailed comparison of numerical flow predictions in cerebral aneurysms using different CFD software. In: Vad J, editor. 15th Conference on Modelling Fluid Flow Budapest, Hungary: ISBN 978-963-08-4588-5 2012: 128-135.

[7] Bernardini A, Larrabide I, Petrini L, et al. Deployment of self-expandable stents in aneurysmatic cerebral vessels: comparison of different computational approaches for interventional planning. Comput Methods Biomech Biomed Eng 2012; 15: 303-311.

[8] Bordás R, Seshadhri S, Janiga G, Skalej M, Thévenin D. Experimental validation of numerical simulations on a cerebral aneurysm phantom model. Interv Med Appl Sci 2012; 4: 193-205.

[9] Burleson AC, Turitto VT. Identification of quantifiable hemodynamic factors in the assessment of cerebral aneurysm behavior. On behalf of the Subcommittee on Biorheology of the Scientific and Standardization Committee of the ISTH. Thromb Haemostasis 1996; 76: 118-123.

[10] Casey M, Wintergerste T. Special Interest Group on "Quality and Trust in Industrial CFD” Best Practice Guidelines. ERCOFTAC 2000.

[11] Cebral JR, Mut F, Raschi M, et al. Aneurysm rupture following treatment with flow-diverting stents: computational hemodynamics analysis of treatment. Am J Neuroradiol 2011; 32: 27-33.

[12] Cebral JR, Mut F, Weir J, Putman C. Quantitative characterization of the hemodynamic environment in ruptured and unruptured brain aneurysms. Am J Neuroradiol 2011; 32: 145-151.

[13] Chen J, Lu XY. Numerical investigation of the non-Newtonian pulsatile blood flow in a bifurcation model with a non-planar branch. J Biomech 2006; 39: 818-832.

[14] Dhar S, Tremmel M, Mocco J, et al. Morphology parameters for intracranial aneurysm rupture risk assessment. Neurosurgery 2008; 63: 185-196.

[15] Ding YH, Lewis DA, Kadirvel R, Dai D, Kallmes DF. The Woven EndoBridge: a new aneurysm occlusion device. Am J Neuroradiol 2011; 32: 607-611.
[16] Gijsen FJ, Allanic E, van de Vosse FN, Janssen JD. The influence of the non-Newtonian properties of blood on the flow in large arteries: unsteady flow in a 90 degrees curved tube. J Biomech 1999; 32: 705-713.

[17] Ha SW, Jang SJ. Clinical analysis of giant intracranial aneurysms with endovascular embolization. J Cerebrovasc Endovasc Neurosurg 2012; 14: 22-28.

[18] Hoi Y, Meng H, Woodward SH, et al. Effects of arterial geometry on aneurysm growth: three-dimensional computational fluid dynamics study. J Neurosurg 2004; 101: 676-681.

[19] Hoi Y, Woodward SH, Kim M, Taulbee DB, Meng H. Validation of CFD simulations of cerebral aneurysms with implication of geometric variations. J Biomed Eng 2006; 128: 844-851.

[20] Janiga G, Beuing O, Seshadhri S, et al. Virtual stenting using real patient data. In: Vad J, editor. 14th Conference on Modelling Fluid Flow, Budapest, Hungary: ISBN 978-963420-987-4 2009: 111-117.

[21] Janiga G, Rössl C, Skalej M, Thévenin D. Realistic virtual intracranial stenting and computational fluid dynamics for treatment analysis. J Biomech 2013; 46: 7-12.

[22] Kim M, Taulbee DB, Tremmel M, Meng H. Comparison of two stents in modifying cerebral aneurysm hemodynamics. Ann Biomed Eng 2008; 36: 726-741.

[23] Lanzino G, Wakhloo AK, Fessler RD, Hartney ML, Guterman LR, Hopkins LN. Efficacy and current limitations of intravascular stents for intracranial internal carotid, vertebral, and basilar artery aneurysms. J Neurosurg 1999; 91: 538-546.

[24] Liepsch DW. Flow in tubes and arteries - a comparison. Biorheology 1986; 23: 395-433.

[25] Liou TM, Liou SN. Pulsatile flows in a lateral aneurysm anchored on a stented and curved parent vessel. Exp Mech 2004; 44: 253-260.

[26] Löhner R, Cebral J, Soto O, Yim P, Burgess JE. Applications of patient-specific CFD in medicine and life sciences. Int J Numer Methods Fluids 2003; 43: 637-650.

[27] Marzo A, Singh P, Larrabide I, et al. Computational hemodynamics in cerebral aneurysms: the effects of modeled versus measured boundary conditions. Ann Biomed Eng 2011; 39: 884-896.

[28] Morales HG, Kim M, Vivas EE, et al. How do coil configuration and packing density influence intra-aneurysmal hemodynamics? Am J Neuroradiol 2011; 32: 1935-1941.

[29] Neugebauer M, Janiga G, Zachow S, Skalej M, Preim B. Generierung qualitativ hochwertiger Modelle für die Simulation von Blutfluss in zerebralen Aneurysmen. In: Hauser H, Straßburger S, Theisel H, editors. Simulation and visualization. Magdeburg, Germany: SCS Publishing House e.V 2008: 221-236.

[30] Owen SJ, White DR. Mesh-based geometry. Int J Numer Methods Eng 2003; 58: 375-395.

[31] Radaelli AG, Augsburger L, Cebral JR, et al. Reproducibility of haemodynamical simulations in a subject-specific stented aneurysm model - a report on the Virtual Intracranial Stenting Challenge 2007. J Biomech 2008; 41: 2069-2081.

[32] Ryu CW, Kwon OK, Koh JS, Kim EJ. Analysis of aneurysm rupture in relation to the geometric indices: aspect ratio, volume, and volume-to-neck ratio. Neuroradiology 2011; 53 : 883-889. 
[33] Schneiders JJ, Vanbavel E, Majoie CB, Ferns SP, van den Berg R. A flow-diverting stent is not a pressure-diverting stent. Am J Neuroradiol 2013; 34: E1-E4.

[34] Schöberl J. NETGEN: an advancing front 2D/3D-mesh generator based on abstract rules. Comput Visual Sci 1997; 1: 41-52.

[35] Schumann C, Neugebauer M, Bade R, Preim B, Peitgen H-O. Implicit vessel surface reconstruction for visualization and CFD simulation. Int J Comput Assist Radiol and Surg 2008; 2: 275-286.

[36] Seshadhri S, Janiga G, Beuing O, Skalej M, Thevenin D. Impact of stents and flow diverters on hemodynamics in idealized aneurysm models. J Biomech Eng 2011; 133: 071005.

[37] Sforza DM, Putman CM, Cebral JR. Hemodynamics of cerebral aneurysms. Annu Rev Fluid Mech 2009; 41: 91-107.

[38] Sforza DM, Putman CM, Scrivano E, Lylyk P, Cebral JR. Blood-flow characteristics in a terminal basilar tip aneurysm prior to its fatal rupture. Am J Neuroradiol 2010; 31: 1127-1131.

[39] Shobayashi Y, Tanoue T, Tateshima S, Tanishita K. Mechanical design of an intracranial stent for treating cerebral aneurysms. Med Eng Phys 2010; 32: 1015-1024.

[40] Shobayashi Y, Tateshima S, Kakizaki R, Sudo R, Tanishita K, Vinuela F. Intra-aneurysmal hemodynamic alterations by a self-expandable intracranial stent and flow diversion stent: high intra-aneurysmal pressure remains regardless of flow velocity reduction. J Neuro Intervent Surg 2012 [Epub ahead of print].

[41] Steinman DA, Hoi Y, Fahy P, et al. Variability of computational fluid dynamics solutions for pressure and flow in a giant aneurysm: the ASME 2012 Summer Bioengineering Conference CFD challenge. J Biomech Eng 2013; 135: 021015/1-13.

[42] Tanoue T, Tateshima S, Villablanca JP, Vinuela F, Tanishita K. Wall shear stress distribution inside growing cerebral aneurysm. Am J Neuroradiol 2011; 32: 1732-1737.

[43] Ujiie H, Liepsch DW, Goetz M, Yamaguchi R, Yonetani H, Takakura K. Hemodynamic study of the anterior communicating artery. Stroke 1996; 27: 2086-2093; discussion 2094.

[44] Valencia A, Morales H, Rivera R, Bravo E, Galvez M. Blood flow dynamics in patient-specific cerebral aneurysm models: the relationship between wall shear stress and aneurysm area index. Med Eng Phys 2008; 30: 329-340.

[45] Wakhloo AK, Lanzino G, Lieber BB, Hopkins LN. Stents for intracranial aneurysms: the beginning of a new endovascular era? Neurosurgery 1998; 43: 377-379.

[46] Xiang J, Natarajan SK, Tremmel M, et al. Hemodynamicmorphologic discriminants for intracranial aneurysm rupture. Stroke 2011; 42: 144-152. 